\title{
EDITORIAL
}

(0) PROSTATE CANCER

\section{Closing the controversies gap in prostate cancer?}

$\mathrm{N}$ owhere is the issue of overtreatment of indolent tumours and undertreatment of high-risk disease with the potential to metastasize more pertinent than in prostate cancer. Although we have begun to define the different evolutionary stages of this disease-classifying men as either 'high risk' so they can commence immediate radical treatment, or 'low risk' that warrants an active surveillance or tissue-preserving focal therapy strategy-there is still much controversy in the field of urological oncology. This controversy extends to the appropriate classification of high-risk and low-risk definitions of this heterogeneous disease, as well as how to treat men who are elderly and could be undertreated simply because of their chronological age rather than based on comorbidity assessment, tumour characteristics and health status. This situation prompted us to cover the most important challenges of diagnosing and treating prostate cancer, and to highlight the key pitfalls and main areas of progress in this field.

One of the most important themes that this issue illustrates is our lack of ability to estimate life expectancy and risk stratification, as eloquently described by Konety and coauthors. Men older than 65 years are often denied curative therapy for fear of not being able to tolerate aggressive treatments, and yet this population can often benefit from 10 to 15 years of life expectancy with such therapy, so we are in danger of throwing away the baby with the bathwater by incorrect assessment of both risk and treatment tolerability in these men.

Although much has been published in the literature about men with high-risk prostate cancer, no classification system exists to determine the outcomes for these men in order to optimize patient management. Chang and coauthors provide an insightful assessment of these issues, and critically discuss the full arena of clinical trial data from multidisciplinary areas spanning radiotherapy, surgical and medical oncology. Unfortunately, they reveal that scarcity of long-term follow up data, statistical limitations, suboptimal trial end points and the lack of comparative data between studies make data interpretation almost impossible. As is always the case in cancer, placing the data in a clinically meaningful context may provide some answers, and the authors propose trial design improvements and suggest high-priority prospective comparisons to help address these long-standing issues.

About $30 \%$ of men diagnosed with low-risk disease harbour high-grade cancer. Although, in most cases these tumours do not require treatment, close scrutiny is prudent to preclude co-existent high-risk disease, and an active surveillance strategy is warranted. Klotz and Emberton discuss the rationale and current status of active surveillance, and the treatment options for men who require treatment. They highlight why less-invasive approaches that include focal therapy, based on mp-MRI, should be considered. By adopting this approach, it is hoped that the number of patients treated in a screened population to avoid each death will be reduced. Complementing this article, Johnson et al. describe the value of $\mathrm{mp}$-MRI in localized and metastatic prostate cancer, and discuss its role in the detection, staging, and treatment planning of this disease.

For men with advanced-stage prostate cancer that has metastasized to the bone or with skeletal involvement, androgen-deprivation therapy can control (although not cure) this disease. However, the intrinsic nature of tumours and their ability to become refractory to treatment result in high morbidity and the need for surgery and radiotherapy to palliate symptoms of skeletal-related events (SRE). Gartrell and Saad describe the burden of SRE, detail the agents currently used to manage these complications (such as inhibitors of the androgen receptor [AR] pathway), and provide an update of the recent promising data related to improved outcomes in men with metastatic castration-resistant prostate cancer (CRPC).

Undoubtedly, some of the most impressive progress in the field of prostate cancer has been made in the area of next-generation AR targeting. In their article, Wong et al. take us through the discovery and evolution of AR therapeutics, and then provide a more in-depth discussion of AR inhibition in CRPC. Understanding the mechanisms of action of targeting the AR in the context of an ever changing disease has been critical in the development of agents that have provided considerable clinical advances. As always, one significant limitation is how we combat resistance to continued blocking of the AR axis. However, early preclinical evidence and emerging clinical data show optimism in this area, which continues to be at the forefront of ongoing and future trials.

In the future, continued molecular characterization of patients will enhance our understanding of the mechanisms of resistance to allow better selection of patients for specific treatments. Despite these controversies in the field of prostate cancer, we are starting to close the gap.

doi:10.1038/nrclinonc.2014.87

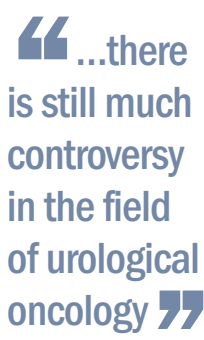

Lisa Hutchinson is the Chief Editor of Nature Reviews Clinical Oncology.

Competing interests The author declares no competing interests. 